\title{
Co-variance nexus between COVID-19 mortality, humidity, and air quality index in Wuhan, China: New insights from partial and multiple wavelet coherence
}

\author{
Zeeshan Fareed $^{1} \cdot$ Najaf Iqbal $^{2,3} \cdot$ Farrukh Shahzad $^{4}$ (1) $\cdot$ Syed Ghulam Meran Shah ${ }^{5} \cdot$ Bushra Zulfiqar $^{6}$. \\ Khurram Shahzad $^{7}$. Shujahat Haider Hashmi ${ }^{8}$ • Umar Shahzad ${ }^{9}$
}

Received: 1 May 2020 / Accepted: 27 May 2020 / Published online: 8 June 2020

(C) Springer Nature B.V. 2020

\begin{abstract}
The worldwide outbreak of COVID-19 disease has caused immense damage to our health and economic and social life. This research article helps to determine the impact of climate on the lethality of this disease. Air quality index and average humidity are selected from the family of climate variables, to determine its impact on the daily new cases of COVID-19-related deaths in Wuhan, China. We have used wavelet analysis (wavelet transform coherence (WTC), partial (PWC), and multiple wavelet coherence (MWC), due to its advantages over traditional time series methods, to study the co-movement nexus between our selected data series. Findings suggest a notable coherence between air quality index, humidity, and mortality in Wuhan during a recent outbreak. Humidity is negatively related to the COVID-19-related deaths, and bad air quality leads to an increase in this mortality. These findings are important for policymakers to save precious human lives by better understanding the interaction of the environment with the COVID-19 disease.
\end{abstract}

Keywords COVID-19 mortality $\cdot$ Partial and multiple wavelet coherence $\cdot$ Air quality index $\cdot$ Humidity $\cdot$ Wuhan

\section{Introduction}

Wuhan, the capital and the largest city of Hubei province, China, was the first major city detected for the novel corona-

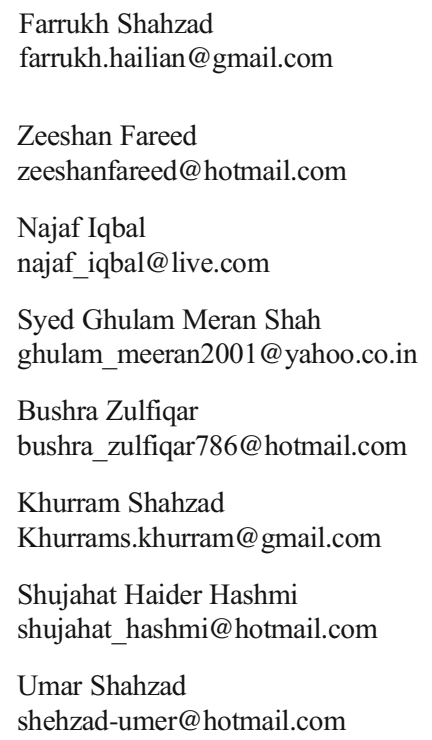

virus disease, namely COVID-19 (Zhou et al. 2020). The first confirmed case of COVID-19 in Wuhan, China was noticed in late 2019. Since then, although the Chinese government took some serious and bold steps of shutting down the road, rail,

2 College of Economics and Management, Hunan University of Arts and Science, Changde, China

3 School of Management, Wuhan University of Technology, Wuhan, China

4 School of Economics and Management, Guangdong University of Petrochemical Technology, Guangdong, China

5 School of Business Administration, Southwestern University of Finance and Economics, Chengdu, China

6 School of Economics, Southwestern University of Finance and Economics, Chengdu, China

7 Key Laboratory of Resource Biology and Biotechnology in Western China, Ministry of Education, College of Life Sciences, Northwest University, Xi'an, People's Republic of China

8 School of Economics, Huazhong University of Science and Technology, Wuhan, Hubei, China

9 School of Economics, Shandong University, Jinan, Shandong Province, People's Republic of China 
Fig. 1 Daily new COVID-19 deaths in Wuhan

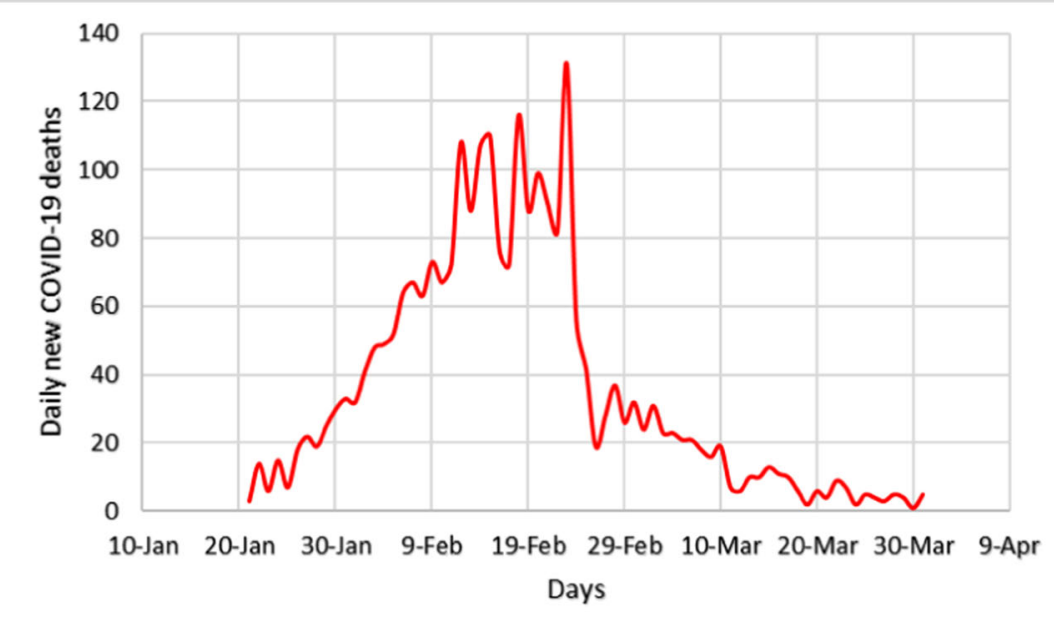

and air transports and closing industries in the whole province, to tackle this deadly COVID-19 virus, still the spread across the entire country could not be stopped (Du et al. 2020).

As of 17 April 2020, the Chinese National Health Commission has reported more than 82,692 confirmed cases and over 4,632 casualties all over the country. Figure 1 shows the time trend of daily new COVID-19 deaths in Wuhan. In addition to China, other countries are also badly affected by this virus, and it has become an acute public health issue worldwide (Zhu et al. 2020). Consequently, as of 30 April 2020, the data from World Health Organization (WHO) shows that more than three million people are infected, and 217,896 have died worldwide due to this pandemic (World Health Organization 2020a). Figure 2 is indicating a map of the Wuhan epidemic on 31 March 2020.

COVID-19 is a new type of coronavirus similar to the severe acute respiratory syndrome coronavirus (SARS$\mathrm{CoV}$ ) which is highly contagious. Due to the widespread transmission of the COVID-19, the WHO officially declared an emergency on March 11, 2020 (World Health Organization 2020b). Generally, COVID-19 patients exhibit common symptoms, including sore throat, cough, high fever, myalgia, and fatigue (Huang et al. 2020). The transmission and spread of COVID-19 may be affected by numerous factors, including medical care quality, climate condition (humidity, temperature, and air quality, etc.), and population density (Wang et al. 2020b). Therefore, it is essential to observe and understand the relationship between environmental factors and the transmission of COVID-19. Figure 3 demonstrates the air quality index which is based on a number of variables $\left(\mathrm{O}_{3}+\mathrm{SO}_{2}+\right.$ $\left.\mathrm{NO}_{2}+\mathrm{PM}_{2.5}+\mathrm{PM}_{10}+\mathrm{CO}\right)$.

Previous research shows that cold and dry weather are instrumental in the spread and survival of dropletmediated contagious diseases, like flu (Shaman and Kohn 2009; Li 2011). Figure 4 shows the time trend of daily, hourly averaged humidity graph in Wuhan.
A recent study checked the impact of humidity and temperature on COVID-19 mortality in Wuhan (Ma et al. 2020). However, our study is different in many aspects; first, we have used the revised data of COVID-19 deaths of Wuhan city (Wuhan revised the numbers of COVID-19 cases and fatalities on 17 April 2020, China Global Television Network (CGTN) reported ${ }^{1}$ ). Second, we employed a novel methodology that extracts events localized in time. The Wavelet technique is robust to the abnormalities in data and determines bidirectional (positive and negative) co-movements at the same time in different time-frequency frames. Third, another difference lies in the humidity-related observations; where other studies are using maximum and minimum values to calculate average humidity (Zhu and Xie 2020; Zhu et al. 2020), we have collected hourly observations and then converted into daily averages. Finally, yet another unique feature is that we have combined air quality index with humidity for studying COVID-19 mortality which is absent in other studies of this kind.

\section{Literature review}

Initially detected in the Chinese city of Wuhan, COVID-19 has spread like a fire across the world due to the fast mobility and advanced communication infrastructure available in the modern-day world (Huang et al. 2020). It is an acute respiratory disease with a highly contagious nature. On average, a patient can infect 2-5 other persons which is a way higher than seasonal influenza and even the SARS outbreak of 2003 (Shao et al. 2020). The COVID-19 infection causes influenza-like symptoms in the majority of the patients.

\footnotetext{
${ }^{1}$ The total COVID-19 confirmed cases were revised up by 325 to 50,333 and the number of deaths up by 1290 to 3869 . The revised figure raised China's overall COVID-19 confirmed cases to 82,692 and death toll to 4,632. The revisions were made by the Wuhan municipal headquarters as per rules and regulations of being responsible for history, the people, and the dead.
} 
Fig. 2 Map showing confirmed cases in Hubie, Province.

COVID-19 Map on 31

March 2020

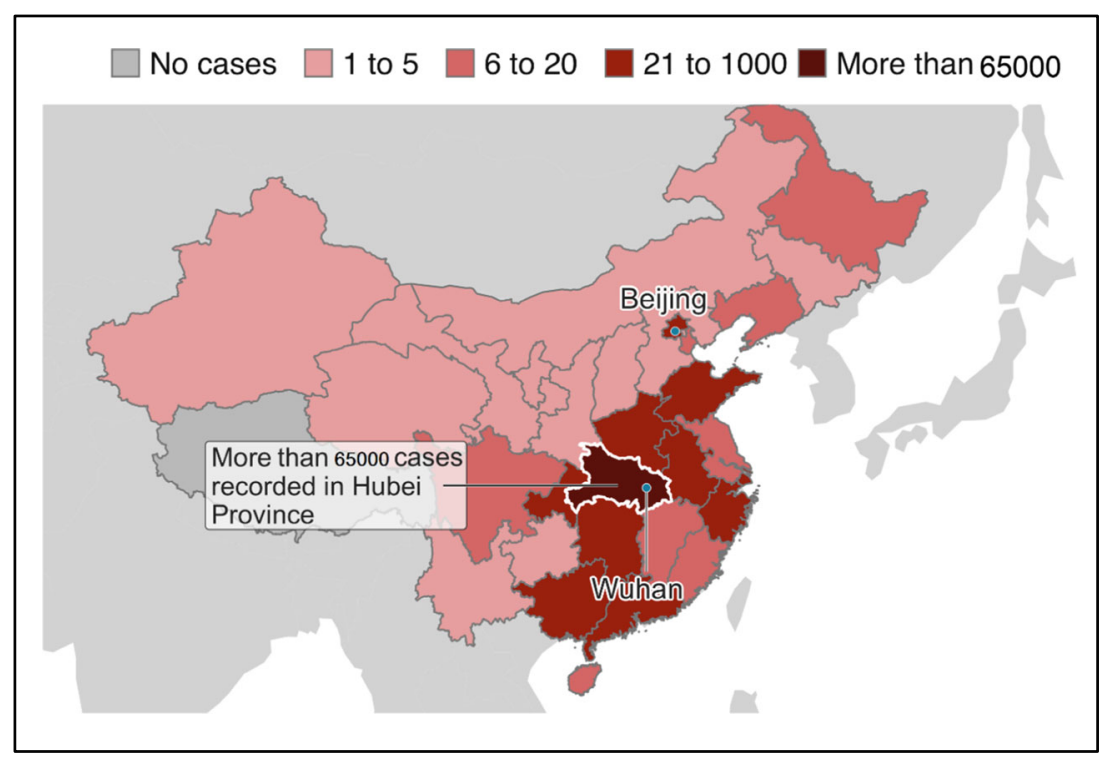

Since pollution and related climatic factors play an important role in the transmission and lethality of respiratory diseases, so, air quality index and humidity can have a fair part in that role too, that may be instrumental in COVID-19 deaths as a result (Wong et al. 2009).

A recent study in South Korea found a significant influence of weather, especially temperature and humidity on several influenza cases; higher relative humidity caused more infections (Park et al. 2020). The role of temperature in containing COVID-19 is mixed while patients should be provided with a comfortable environment for faster recoveries (Iqbal et al. 2020; Shahzad et al. 2020).

Since COVID-19 is caused by a novel virus that belongs to the corona-virus family, it may behave like SARS when it comes to different atmospheric conditions, including humidity and air quality index (AQI). Casanova et al. (2010) emphasized that SARS corona-virus could survive for longer on surfaces at average relative humidity and typical room temperature (with air-conditioning). SARS corona-virus could survive on smooth surfaces for longer at a higher relative humidity (Chan et al. 2011).

Prolonged life on surfaces results in an increased number of infections and faster spread. A recent study involving Italy, the USA, and China, on the relationship between AQI and COVID-19 outbreak finds that the level of CO (carbon monoxide) and $\mathrm{SO}_{2}$ (sulphur dioxide) in the air, is positively related with the number of infections, and lower air quality is causing more deaths (Pansini and Fornacca 2020). This implies that areas with poor air quality may be more vulnerable to severe COVID-19 outbreaks. Another study on 72 cities of China, indicates that an increase in $\mathrm{PM}_{2.5}$ and $\mathrm{PM}_{10}$ particles in the air leads to a higher number of daily COVID-19 infections, even after controlling for the absolute humidity (Wang et al. 2020a). This implies that more pollution leads to a higher
Fig. 3 Daily air quality index in Wuhan

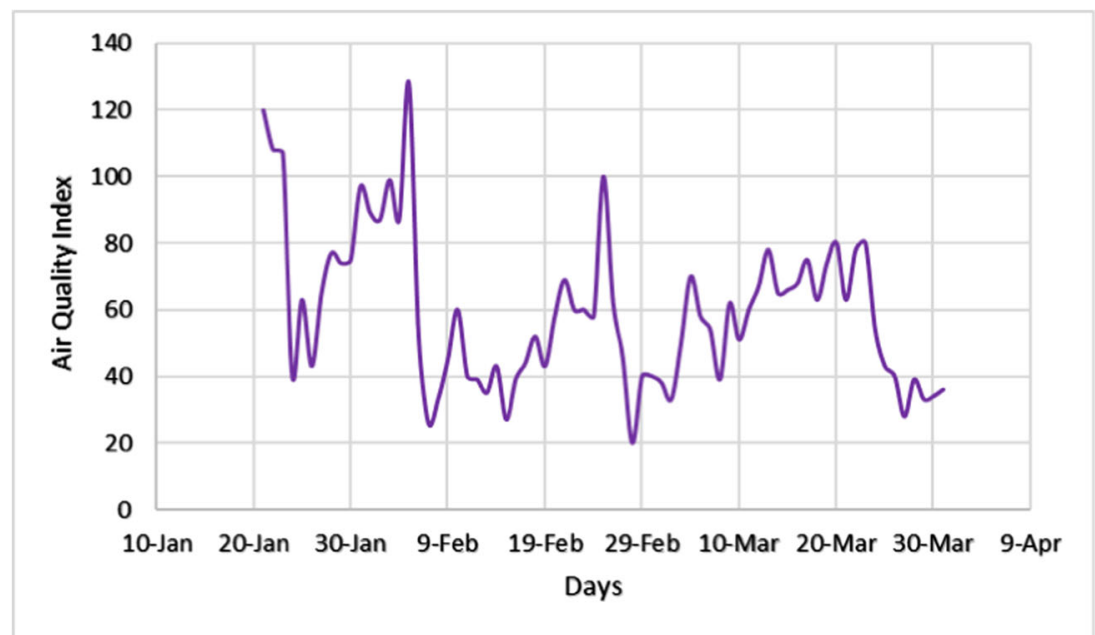


Fig. 4 Daily hourly average humidity $(\%)$ in Wuhan

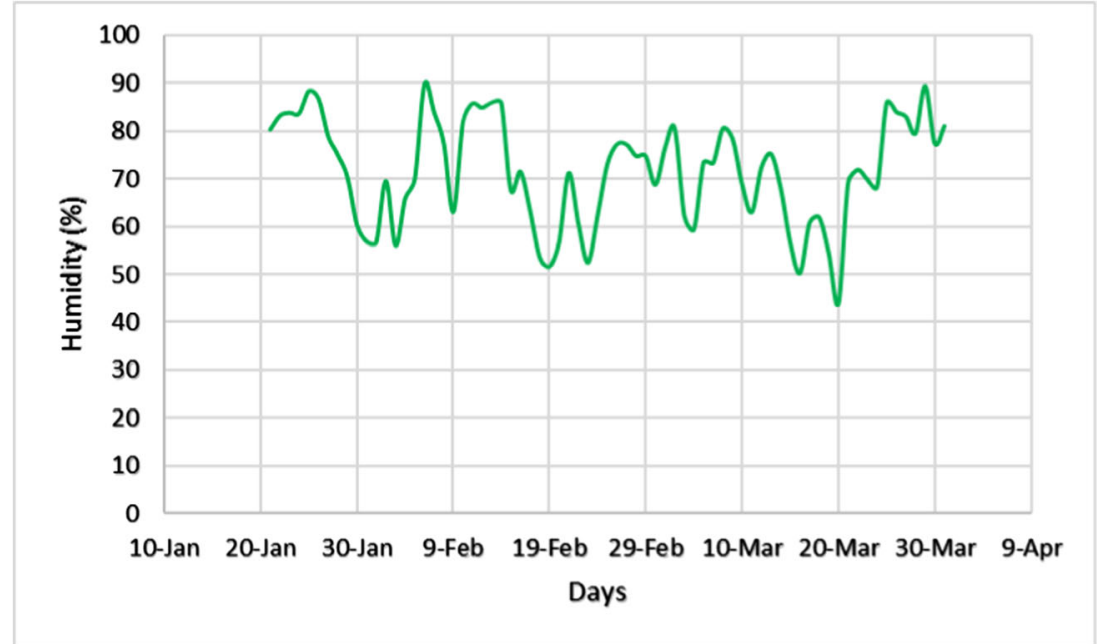

number of COVID-19 infections and faster spread in China. Similar results are reported for Italy where a high level of $\mathrm{PM}_{10}$ airborne particles in the northern part seems to have played a role in the fast spread of COVID-19 (Setti et al. 2020). An increase in humidity is negatively related to the doubling time of COVID-19 infections in the whole of China (Oliveiros et al. 2020).

The spread of COVID-19 in Jakarta, the capital city of Indonesia, is found to be correlated with the weather, especially temperature and humidity (Tosepu et al. 2020). A study on 63 cities of China suggests that a higher level of $\mathrm{NO}_{2}$ (a component of AQI) in the air might have led to increasing the $R^{0}$ (spread-ability) of COVID-19 (Yao et al. 2020). As air pollution can cause a number of respiratory problems, a study on 120 cities of China relates air pollution with COVID-19 outbreak and finds majority of the components of air pollution $\left(\mathrm{PM}_{2.5}, \mathrm{PM}_{10}, \mathrm{NO}_{2}, \mathrm{NO}_{3}\right)$ linked positively with the COVID19 infections (Zhu et al. 2020). Air pollution overall, while $\mathrm{PM}_{10}$ and $\mathrm{PM}_{2.5}$ specifically, are linked with the millions of deaths in the world annually by causing acute respiratory diseases, like asthma and lungs inflammation (Lelieveld et al. 2015, 2019). A meta-analysis study found that outdoor air pollution is significantly related to asthma and respiratory diseases (Anderson et al. 2013). The particles $\mathrm{PM}_{2.5}$ and $\mathrm{O}_{3}$ are found to be significantly related to premature deaths and acute health issues according to a study in the USA (Fann and Risley 2013). These studies imply that COVID-19 patients may find it more difficult to recover in case of bad air quality (Contini 2020).

A study on the latitude analysis and community outbreaks of COVID-19 around the world finds that low levels of specific and relative humidity are positively linked with significant community outbreaks (Sajadi et al. 2020). This is consistent with many other studies mentioned above, suggesting that an increase in humidity can lead to a decrease in COVID-19 cases.
All of these mentioned studies show that air quality and humidity are significantly linked with the speed of COVID-19 spread. Most of these authors report a positive link of COVID19 spread with poor air quality and negative with an increase in humidity. However, we have employed a different methodology with several advantages over traditional time series models and econometric techniques to study this relationship.

\section{Data and methodology}

\section{Data}

Daily new deaths due to COVID-19 for the Wuhan city, between 21 January 2020 and 31 March 2020 were collected from the reports of the official website of the Chinese National Health Commission (CNHC, http://www.nhc.gov. $\mathrm{cn} /$ ). Daily data of air pollution index of Wuhan city were manually obtained from an online platform (https://www. aqistudy.cn), analyzing and monitoring the air quality based on six factors including carbon monoxide $(\mathrm{CO})$, ozone $\left(\mathrm{O}_{3}\right)$, sulfur dioxide $\left(\mathrm{SO}_{2}\right)$, nitrogen dioxide $\left(\mathrm{NO}_{2}\right)$, particles with diameters $\leq 10 \mu \mathrm{m}\left(\mathrm{PM}_{10}\right)$, and particles with diameters $\leq 2$. $5 \mu \mathrm{m}\left(\mathrm{PM}_{2.5}\right)$. The humidity data of Wuhan was collected on an hourly basis and then manually converted into daily averages, obtained from (https://www.wunderground.com).

\section{Methodology}

We have used the wavelet methodology for our time series analysis. Specifically, we employed wavelet transform coherence (WTC), partial wavelet coherence (PWC), and multiple wavelet coherence (MWC) for the detection of the pattern of co-movements and associations in our data series. The use of wavelet analysis allows us to analyze the non-normal and nonstationary time series. We employed this methodology 
because of its least assumptions and ability to analyze localized interactions from a time-frequency perspective.

The two main types of wavelet transform are discrete wavelet transform and continuous wavelet transform. We have used continuous wavelet transform (CWT) due to its suitability here in our data set. Multiple wavelet functions have been developed according to the nature of variability in a data set. We have used "Morlet wavelet," which is also the most extensively used wavelet function among others. Monte Carlo method helps to determine the level of significance here. The detailed wavelet methodology has been adopted from earlier studies (Ng and Chan 2012; Aloui et al. 2018; Hkiri et al. 2018).

The WTC determines the direction and strength of the association between two series $x_{1}$ and $x_{2}$ by extracting localized time-frequency events. The cone of influence (COI) splits the whole wavelet power spectrum into dark and light shades, providing significant edge effects. The values beyond the COI indicate the significance level of each scale in the wavelet coherence (Torrence and Compo 1998). The following is the mathematical equation of wavelet transforms coherence:

$R^{2}(m, n)=\frac{\mid N\left(\left.N^{-1} W_{x y}(m, n)\right|^{2}\right.}{N\left(N^{-1}\left|W_{x}(m, n)\right|^{2} N\left(N^{-1}\left|W_{y}(m, n)\right|^{2}\right.\right.}$

The values of wavelet coherence remain between 0 and 1 , $0 \leq R^{2}(m, n) \leq 1$, where zero is the indication of no coherence while 1 represents the perfect coherence between the variables.

\section{Partial wavelet coherence (PWC)}

The partial wavelet coherence follows the concept of simple correlation. The PWC determines the co-movement between two series after canceling the effects of another confounder variable. The Eqs. 2 to 7 represent the co-movements (WTC) between different combinations of $x_{1}, x_{2}$, and $y$.

$$
\begin{aligned}
& R\left(x_{1}, x_{2}\right)=\frac{S\left[W\left(x_{1}, x_{2}\right)\right]}{\sqrt{S\left[W\left(x_{1}\right)\right] S\left[W\left(x_{2}\right)\right]}} \\
& R^{2}\left(x_{1}, x_{2}\right)=R\left(x_{1}, x_{2}\right) \cdot R\left(x_{1}, x_{2}\right)^{*} \\
& R\left(x_{1}, y\right)=\frac{S\left[W\left(x_{1}, y\right)\right]}{\sqrt{S\left[W\left(x_{1}\right)\right] S[W(y)]}} \\
& R^{2}\left(x_{1}, y\right)=R\left(x_{1}, y\right) \cdot R\left(x_{1}, y\right)^{*} \\
& R\left(x_{2}, y\right)=\frac{S\left[W\left(x_{2}, y\right)\right]}{\sqrt{S\left[W\left(x_{2}\right)\right] S[W(y)]}} \\
& R^{2}\left(x_{2}, y\right)=R\left(x_{2}, y\right) \cdot R\left(x_{2}, y\right)^{*}
\end{aligned}
$$

The following equation shows the PWC between two variables while cancelling the effects of another (third) confounder variable.

$\operatorname{RP}^{2}\left(y, x_{1}, x_{2}\right)=\frac{\left|R\left(y, x_{1}\right)-R\left(y, x_{2}\right) \cdot R\left(y, x_{1}\right) *\right|^{2}}{\left[1-R\left(y, x_{2}\right)\right]^{2}\left[1-R\left(x_{2}, x_{1}\right)\right]^{2}}$

\section{Multiple wavelet coherence (MWC)}

The MWC enables us to study nexus between more than two variables at the same time. Actually, it calculates the combined effects of the independent variables on a dependent variable at varying time-frequency spaces. The following Eq. (9) presents the mathematical expression of multiple wavelet coherence.

$$
\begin{aligned}
& R M^{2}\left(y, x_{2}, x_{1}\right) \\
& \quad=\frac{R^{2}\left(y, x_{1}\right)+R^{2}\left(y, x_{2}\right)-2 R \mathrm{e}\left[R\left(y, x_{1}\right) \cdot R\left(y, x_{2}\right) * \cdot R\left(x_{2}, x_{1}\right) *\right]}{1-R^{2}\left(x_{2}, x_{1}\right)} ;
\end{aligned}
$$

The use of wavelet methodology has unique features as compared with traditional time series techniques. We can analyze non-stationary and non-normally distributed data efficiently. The most significant advantage of this method is its ability to identify competing (positive and negative) types of the association at the same time. We can check if the variables are positively correlated at one time-frequency spot and negatively correlated with others. In this way, we can extract every localized interaction and co-movement in multiple series of data. This feature is unique as compared to traditional correlation and regression models which only tell us about an overall average relationship during the whole observation period.

Due to the abovementioned advantages, this method can give us better results and a good understanding of the phenomenon under observation. That is why it is extensively used in modeling signal processing and oceanic technology.

Table 1 shows the summary statistics and correlation between observed variables. The average number of daily deaths stands at 36, ranging from a minimum of 1 to a maximum of 131. Daily humidity ranges from 44.08 to $89.58 \%$ while averaging at $71.51 \%$. The minimum and maximum value of AQI remained 20 and 128 respectively, averaging at 59.56. The AQI shows a significant and strong negative relationship with both humidity $(-0.61)$ and COVID-19 $(-0.70)$. The humidity and COVID-19 are also negatively correlated $(-0.51)$ but the coefficient is insignificant.

Figure 5a represents COVID-19 deaths in the city of Wuhan after continuous wavelet transform. Red color means more variation while the blue shows no significant variation. 
Table 1 Descriptive statistics

\begin{tabular}{llll}
\hline Variable & $\begin{array}{l}\text { COVID- } \\
19\end{array}$ & HUM & AQI \\
\hline Mean & 35.958 & 71.519 & 59.563 \\
Std. dev. & 34.147 & 11.067 & 23.273 \\
Min & 1 & 44.083 & 20 \\
Max & 131 & 89.583 & 128 \\
Jarque-Bera & 12.92 & 8.520 & 7.376 \\
$p$ value & 0.001 & 0.013 & 0.025 \\
Correlation matrix & & & \\
COVID-19 & 1 & 1 & \\
HUM & -0.511 & & \\
& 0.000 & $-0.705^{*}$ & \\
AQI & $-0.610^{*}$ & 0.000 & \\
& 0.000 & & \\
\hline
\end{tabular}

*Shows the $5 \%$ level of significance

Numbers along the y-axis represent different frequencies while the $\mathrm{x}$-axis shows time. The red island-shaped areas inside the black lining can be seen in front of frequency bands of $0-4$ and $4-8$, during the third and fourth weeks of observation. This shows a significant variation in deaths during the given time period.

Figure $5 b$ shows the result of the CWT of daily average percentage of humidity in Wuhan. Two red-colored islands with black outlines are fairly visible, one small-circular while other long-oval shaped in the frequency range of $0-4$ during the third and seventh-eighth weeks of observation, respectively.

Figure $5 \mathrm{c}$ offers a CWT of daily air quality index in Wuhan. Two prominent dark red colored oval shapes with thick black lining show significant variations in the frequency domain of 0-4 and 4-8 during the second and third weeks of the observation period. Another small dark red-colored circle is also present on the upper left corner of the figure in the frequency domain of $0-4$ during the first week of observation.

Figure 6a shows the WTC between COVID-19 deaths and average humidity in Wuhan. A red-colored circular shape can be observed in front of the frequency band of 4-8 during the fourth week of the observation period. Small arrows' clusters are visible throughout which represent coherence between COVID-19 deaths and average humidity in Wuhan. Left and upwards direction of arrows inside the circular shape means out phase coherence running from humidity to COVID-19 deaths. This implies a negative relationship between humidity and COVID-19 deaths in Wuhan, during this specific timefrequency domain. Another red-colored, thin oval shape is observable in the frequency range of $8-16$ periods during the sixth and seventh weeks of observation. Arrows inside this oval-shape are pointing left and downwards, showing an out
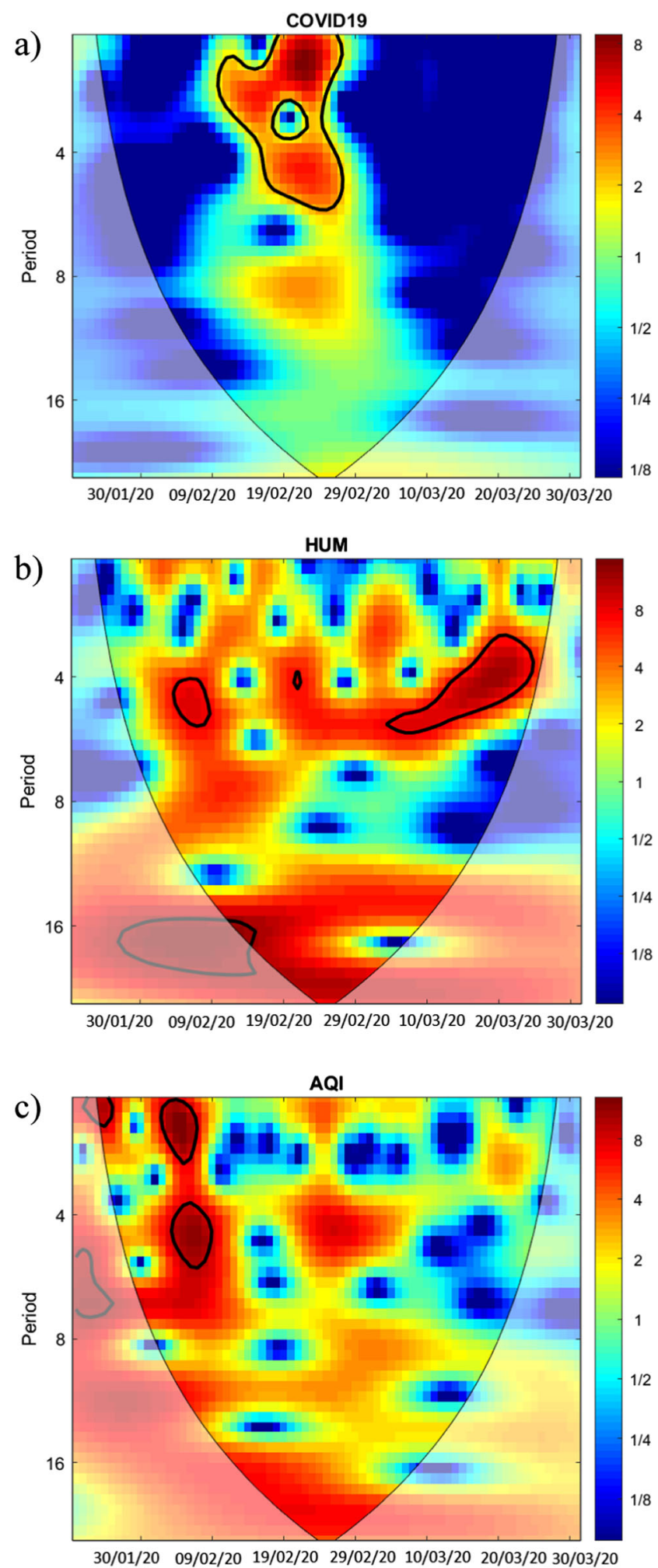

Fig. 5 Continuous wavelet transforms of COVID-19, HUM, and AQI

phase coherence running from COVID-19 deaths to humidity. These results suggest a bi-directional negative relationship between humidity and COVID-19 deaths in Wuhan, during our observation period at different frequency domains. 

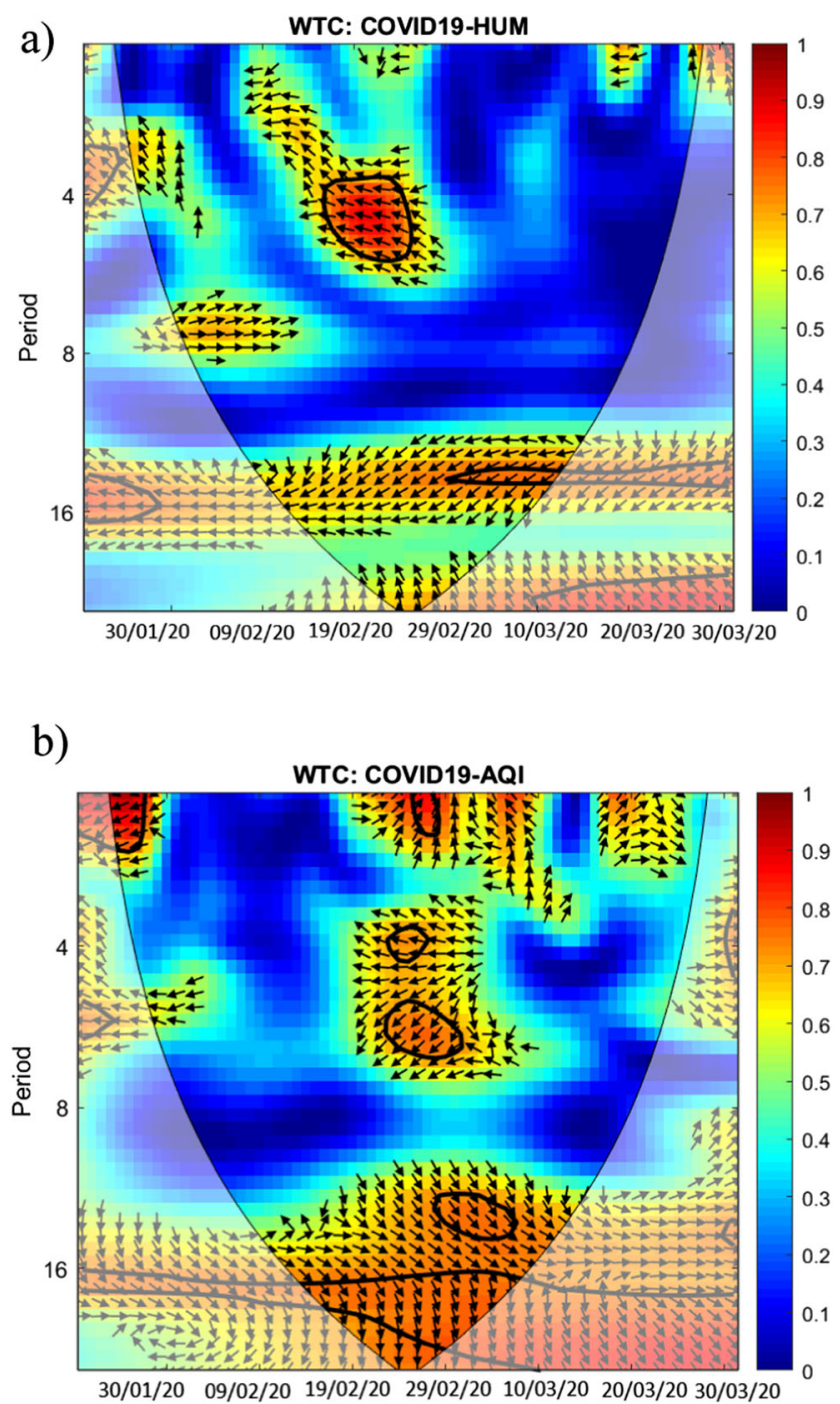

c)

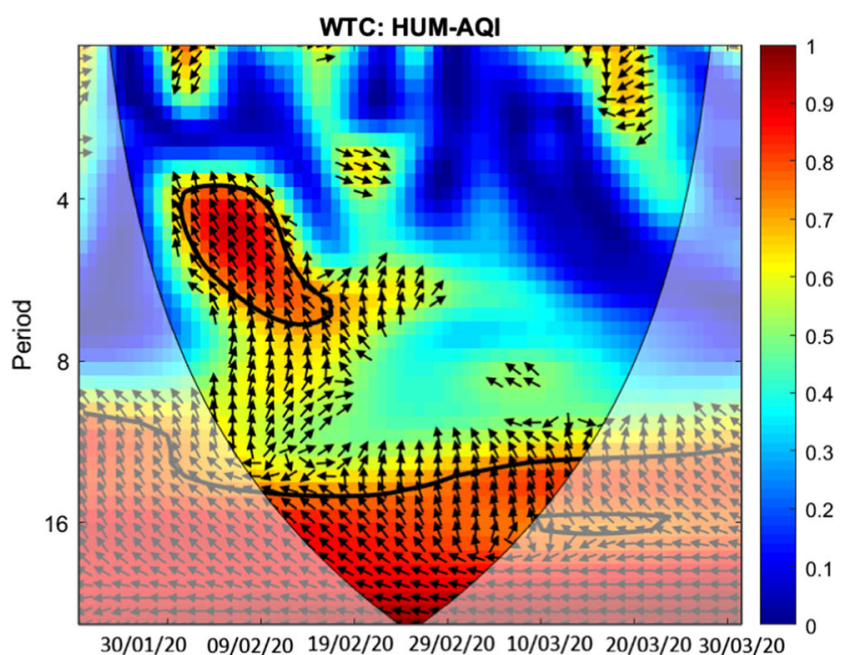

Fig. 6 Wavelet coherence transform of COVID-19, HUM, and AQI
Figure $6 \mathrm{~b}$ shows the WTC between COVID-19 deaths and the air quality index in Wuhan. Few small and red-colored circular shapes can be found scattered throughout the figure. In the frequency band of $0-4$, there are two circles during the first and fifth weeks of observation. Other circles lie in the frequency bands of 4-8, 8-16, and onwards during the fifth and sixth weeks of observation. A large red area on the bottom and a small circle above that, contain arrows pointing right and downwards showing an in phase coherence between AQI and COVID-19 deaths, running from AQI to COVID-19. This implies that an increase in AQI (bad air quality) is leading to more deaths caused by COVID-19 in the long term, remaining within our observation time period. Other circles are showing arrows pointing towards various directions implying a mixed association during those specific time-frequency bands.

Figure $6 \mathrm{c}$ shows the WTC between average humidity and air quality index in Wuhan. Two significant and large red areas are observable inside the black linings. One island is in the frequency band of 4-8 during second to third weeks of observation while the other lies in the frequency range of $8-16$ and onwards during third to seventh weeks in the time domain. The arrows inside these islands are pointing towards left and upwards dominantly, indicating an out phase coherence, running from air quality index to average humidity. This implies a negative relationship between humidity and air quality in Wuhan during our observation period.

Figure 7a shows the results of the PWC, involving COVID19 deaths, average humidity, and air quality index in Wuhan. In simple words, it describes the wavelet coherence between COVID-19 deaths and average humidity after taking out the common effects of air quality index on the relationship between COVID-19 deaths and humidity. Small red areas inside the black linings show the coherence in frequency bands of $0-4$ and 4-8 during the fourth week of observation. If we compare Fig. 7a with the WTC results between COVID-19 deaths and humidity from Fig. 6a, we notice some differences between the two figures. These differences represent that AQI has a significant influence over wavelet coherence (relationship) between humidity and COVID-19 deaths. In simple words, the role of AQI in the association between humidity and COVID-19 deaths can be thought of as a "confounding variable."

If we compare Fig. 7c with an earlier Fig. 6c shows the results of WTC between humidity and air quality, there are not many significant differences. Red-colored islands lie almost at similar time-frequency spots in both of these figures. This implies that COVID-19 deaths have little if any role in the coherence (relationship) between humidity and air quality index in Wuhan.

Figure 7e demonstrates the PWC involving AQI, COVID19 deaths, and humidity is significant because there are visible differences as compared to Fig. 6b showing WTC results between AQI and COVID-19 deaths. This implies that humidity plays a vital role in the coherence (relationship) between AQI and COVID-19 deaths. A few small red-colored islands on the 

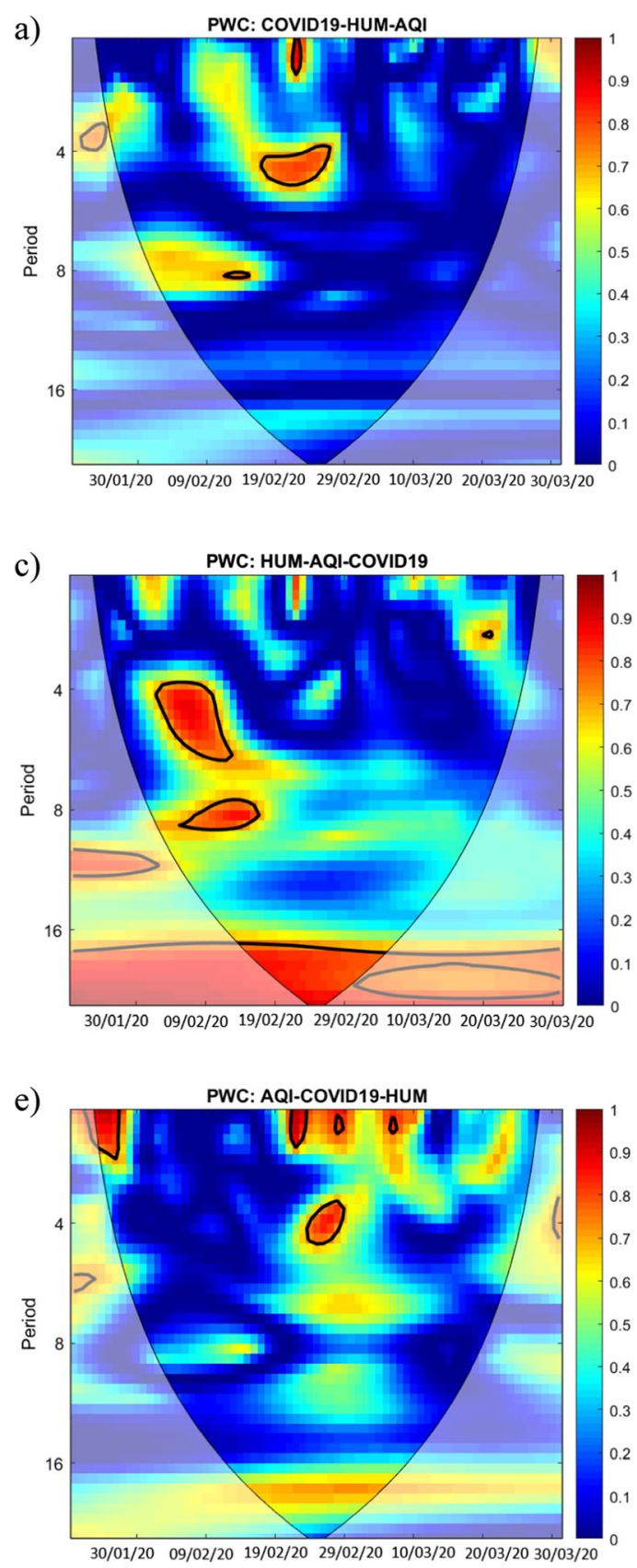

Fig. 7 Partial and multiple wavelet coherence of COVID-19, HUM, and AQI

upper side, in the frequency band of $0-4$ show a significant coherence between AQI and COVID-19 deaths even after controlling for the effects of humidity during the first, fourth, fifth, and sixth weeks of observation.

Figure $7 \mathrm{~b}$ shows the result of the MWC involving COVID19 deaths in Wuhan as a dependent while humidity and AQI as independent variables. Specifically, it tells us how good the combined effect of humidity and AQI predicts COVID-19related deaths in Wuhan. A thin vertical bar on the right side of the figure shows different levels of association tied up with different colors. As it moves from bottom to top (blue to red), the strength of the relationship keeps on increasing, reaching
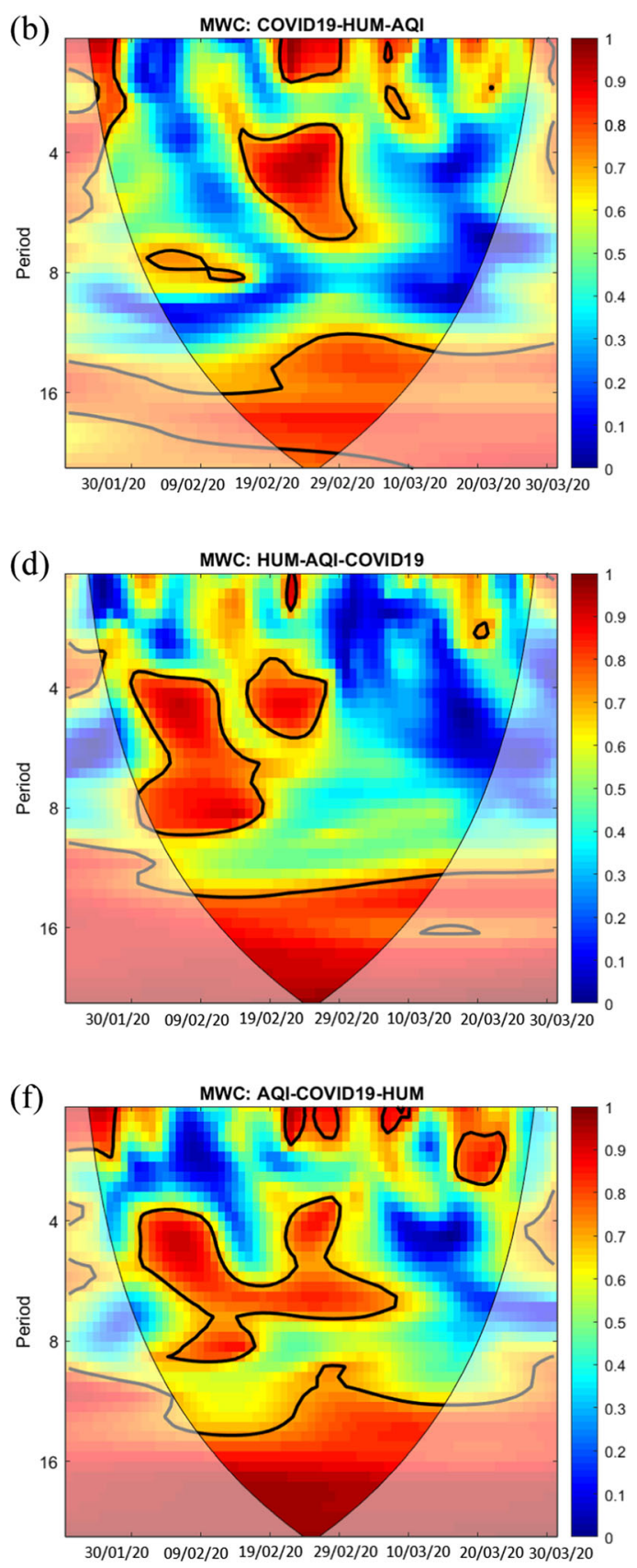

its maximum level of 1 represented by the dark red color. Large and small islands with red color inside and clear black outlining are scattered within different time-frequency combinations. These red shapes show the combined predictive power of humidity and AQI in determining the number of COVID-19-related deaths. As evident from the figure, humidity and AQI show significant MWC with COVID-19 deaths in all frequency bands during different periods of observation. In the middle of the observation period, during the fourth, fifth, and sixth weeks, a large island is found at the bottom of the figure, indicating a significant coherence. Another two islands are prominent on the top of this island during the fourth and 
fifth weeks of observation in the frequency bands of $0-4$ and 4-8. Overall results reveal a significant coherence between humidity-AQI and COVID-19-related deaths in Wuhan.

Figure $7 \mathrm{~d}$ presents MWC results taking humidity as a dependent while AQI and COVID-19 deaths as independent variables. A significant coherence can be observed in frequency bands of 4-8, 8-16, and onwards during the second and fourth, second-third, and third-seventh weeks of observation indicated by large red-colored shapes. Two small islands can also be found in the $0-4$ frequency band during the fourth and eighth weeks of observation.

Figure 7f of MWC shows AQI as a dependent while humidity and COVID-19 deaths as independent variables. In a frequency band of $0-4$, some islands are present during the first, fourth, fifth, sixth, and eighth weeks of observation. More red areas are present in 4-8, 8-16, and onwards frequency bands during the second-sixth, third-seventh, and fourthfifth weeks. Overall results present a noticeable coherence between AQI and the combination of humidity and COVID19 deaths. Dark red color inside these island type shapes can be compared with a level of matching association between 0.80 and 1.00 , according to the vertical bar on the right side.

\section{Conclusion}

The environment played an important role in the COVID-19related deaths in Wuhan. A rise in average humidity leads to a decrease in COVID-19-related deaths as revealed by the WTC at varying time-frequency spots. Deteriorating air quality resulted in more COVID-19 deaths in the medium run during our observation period. The air quality index further had an important role in the negative coherence between humidity and COVID-19 deaths. Bad environment conditions caused more COVID-19-related deaths, not only directly but also indirectly by exerting a negative influence on the role of average humidity. These results imply that COVID-19 patients should be provided with a clean environment for an early and smooth recovery.

Our results are limited to the context of cities with similar weather conditions and containment measures as of Wuhan and future studies should consider a larger dataset including varying weather conditions.

\section{References}

Aloui C, Hkiri B, Hammoudeh S, Shahbaz M (2018) A multiple and partial wavelet analysis of the oil price, inflation, exchange rate, and economic growth nexus in Saudi Arabia. Emerg Mark Financ Trade 54(4):935-956
Anderson HR, Favarato G, Atkinson RW (2013) Long-term exposure to air pollution and the incidence of asthma: meta-analysis of cohort studies. Air Qual Atmos Heal 6:47-56

Casanova LM, Jeon S, Rutala WA, Weber DJ, Sobsey MD (2010) Effects of air temperature and relative humidity on coronavirus survival on surfaces. Appl Environ Microbiol 76:2712-2717. https://doi.org/10. 1128/AEM.02291-09

Chan KH, Peiris JSM, Lam SY, Poon LLM, Yuen KY, Seto WH (2011) The effects of temperature and relative humidity on the viability of the SARS coronavirus. Adv Virol 2011:1-7. https://doi.org/10. $1155 / 2011 / 734690$

Contini D (2020) Does air pollution influence COVID-19 outbreaks?. 1: $19-23$

Du Z, Wang L, Cauchemez S et al (2020) Risk for transportation of 2019 novel coronavirus disease from Wuhan to other cities in China. Emerg Infect Dis 26:1049-1052. https://doi.org/10.3201/eid2605. 200146

Fann N, Risley D (2013) The public health context for PM 2.5 and ozone air quality trends. Air Qual Atmos Heal 6:1-11

Hkiri B, Hammoudeh S, Aloui C, Shahbaz M (2018) The interconnections between US financial CDS spreads and control variables: New evidence using partial and multivariate wavelet coherences. Int Rev Econ Financ 57:237-257

Huang C, Wang Y, Li X, Ren L, Zhao J, Hu Y, Zhang L, Fan G, Xu J, Gu X, Cheng Z, Yu T, Xia J, Wei Y, Wu W, Xie X, Yin W, Li H, Liu M, Xiao Y, Gao H, Guo L, Xie J, Wang G, Jiang R, Gao Z, Jin Q, Wang J, Cao B (2020) Clinical features of patients infected with 2019 novel coronavirus in Wuhan, China. Lancet 395:497-506. https://doi.org/10.1016/S0140-6736(20)30183-5

Iqbal N, Fareed Z, Shahzad F et al (2020) Nexus between COVID-19, temperature and exchange rate in Wuhan City: new findings from partial and multiple wavelet coherence. Sci Total Environ 138916

Lelieveld J, Evans JS, Fnais M, et al (2015) The contribution of outdoor air pollution sources to premature mortality on a global scale. https:// doi.org/10.1038/nature15371

Lelieveld J, Klingmu K, Pozzer A, et al (2019) Cardiovascular disease burden from ambient air pollution in Europe reassessed using novel hazard ratio functions. 1590-1596. https://doi.org/10.1093/ eurheartj/ehz135

Li S (2011) Environmentally mediated transmission models for influenza and the relationships with

Ma Y, Zhao Y, Liu J, He X, Wang B, Fu S, Yan J, Niu J, Zhou J, Luo B (2020) Effects of temperature variation and humidity on the death of COVID-19 in Wuhan, China. Sci Total Environ 724:138226. https://doi.org/10.1016/j.scitotenv.2020.138226

Ng EK, Chan JC (2012) Geophysical applications of partial wavelet coherence and multiple wavelet coherence. J Atmos Ocean Technol 29(12):1845-1853

Oliveiros B, Caramelo L, Ferreira NC, Caramelo F (2020) Role of temperature and humidity in the modulation of the doubling time of COVID-19 cases. medRxiv

Pansini R, Fornacca D (2020) COVID-19 higher induced mortality in Chinese regions with lower air quality

Park JE, Son WS, Ryu Y, Choi SB, Kwon O, Ahn I (2020) Effects of temperature, humidity, and diurnal temperature range on influenza incidence in a temperate region. Influenza Other Respi Viruses 14: 11-18. https://doi.org/10.1111/irv.12682

Sajadi MM, Habibzadeh P, Vintzileos A, Shokouhi S, Miralles-Wilhelm F, Amoroso A (2020) Temperature and latitude analysis to predict potential spread and seasonality for COVID-19. Available at SSRN 3550308

Setti L, Passarini F, de Gennaro G, Di Gilio A, Palmisani J, Buono P, Fornari G, Perrone MG, Piazzalunga A, Barbieri P, Rizzo E, Miani A (2020) Evaluation of the potential relationship between particulate matter (PM) pollution and COVID-19 infection spread in Italy 
Shahzad F, Shahzad U, Fareed Z, et al (2020) Asymmetric nexus between temperature and COVID-19 in the top ten affected provinces of China: a current application of quantile-on-quantile approach. Sci Total Environ 139115

Shaman J, Kohn M (2009) Absolute humidity modulates influenza survival, transmission, and seasonality. Proc Natl Acad Sci U S A 106: 3243-3248. https://doi.org/10.1073/pnas.0806852106

Shao N, Cheng J, Chen W (2020) The reproductive number R 0 of COVID19 based on estimate of a statistical time delay dynamical system. 1-10

Torrence C, Compo GP (1998) A practical guide to wavelet analysis. Bull Am Meteorol Soc 79(1):61-78

Tosepu R, Gunawan J, Effendy DS, Ahmad LOAI, Lestari H, Bahar H, Asfian P (2020) Correlation between weather and Covid-19 pandemic in Jakarta, Indonesia. Sci Total Environ 138436. https://doi. org/10.1016/j.scitotenv.2020.138436

Wang B, Liu J, Fu S, et al (2020a) An effect assessment of airborne particulate matter pollution on COVID-19: a multi-city study in China

Wang J, Tang K, Feng K, Lv W (2020b) High temperature and high humidity reduce the transmission of COVID-19. SSRN Electron J. https://doi.org/10.2139/ssrn.3551767

Wong CM, Yang L, Thach TQ, et al (2009) Modification by influenza on health effects of air pollution in Hong Kong. 248:248-253. https:// doi.org/10.1289/ehp. 11605
World Health Organization (2020a) Coronavirus disease (COVID-19) outbreak situatione, Access Date: 30 April 2020.

World Health Organization (2020b) WHO Director-General's opening remarks at the media briefing on COVID-19-11March 2020

Yao Y, Pan J, Liu Z, Meng X, Wang W, Kan H, Wang W (2020) Ambient nitrogen dioxide pollution and spread ability of COVID19 in Chinese cities. medRxiv

Zhou F, Yu T, Du R et al (2020) Clinical course and risk factors for mortality of adult inpatients with COVID-19 in Wuhan, China: a retrospective cohort study. Lancet 395:1054-1062. https://doi.org/ 10.1016/S0140-6736(20)30566-3

Zhu Y, Xie J (2020) Association between ambient temperature and COVID-19 infection in 122 cities from China. Sci Total Environ 724:138201. https://doi.org/10.1016/j.scitotenv.2020.138201

Zhu Y, Xie J, Huang F, Cao L (2020) Association between short-term exposure to air pollution and COVID-19 infection: evidence from China. Sci Total Environ 727:138704. https://doi.org/10.1016/j. scitotenv.2020.138704

Publisher's note Springer Nature remains neutral with regard to jurisdictional claims in published maps and institutional affiliations. 